\title{
HUMAN IMPLANTABLE WIRELESS MICROSTIMULATORS AND MICROSENSORS
}

\author{
J.H. Schulman, Ph.D.* \\ Alfred E. Mann Foundation for Biomedical Engineering, Valencia, California, USA
}

\begin{abstract}
Stroke, spinal cord injury, and other injuries have today left over 5 million patients with partial or complete paralysis in one or more limbs. These patients often have viable skeletal muscles with attached viable motor neurons, but no way to activate their neuralmuscular system to contract their paralyzed muscles. In 1989 the National Institute of Health $(\mathrm{NIH})$ requested the development of magnetically powered injectable stimulators to restore function to these patients. This request has led to the development of a system of wirelessly coordinated battery powered rechargeable implantable microdevices specifically designed to restore function to these patients with a minimum of surgery, and external equipment.
\end{abstract}

\section{INTRODUCTION}

There have been many attempts to provide function to paralyzed or partially paralyzed limbs using implantable multi-lead stimulators during the last 40 years. Two of these attempts which are no longer on the market are shown in Figure 1 and Figure 1A.

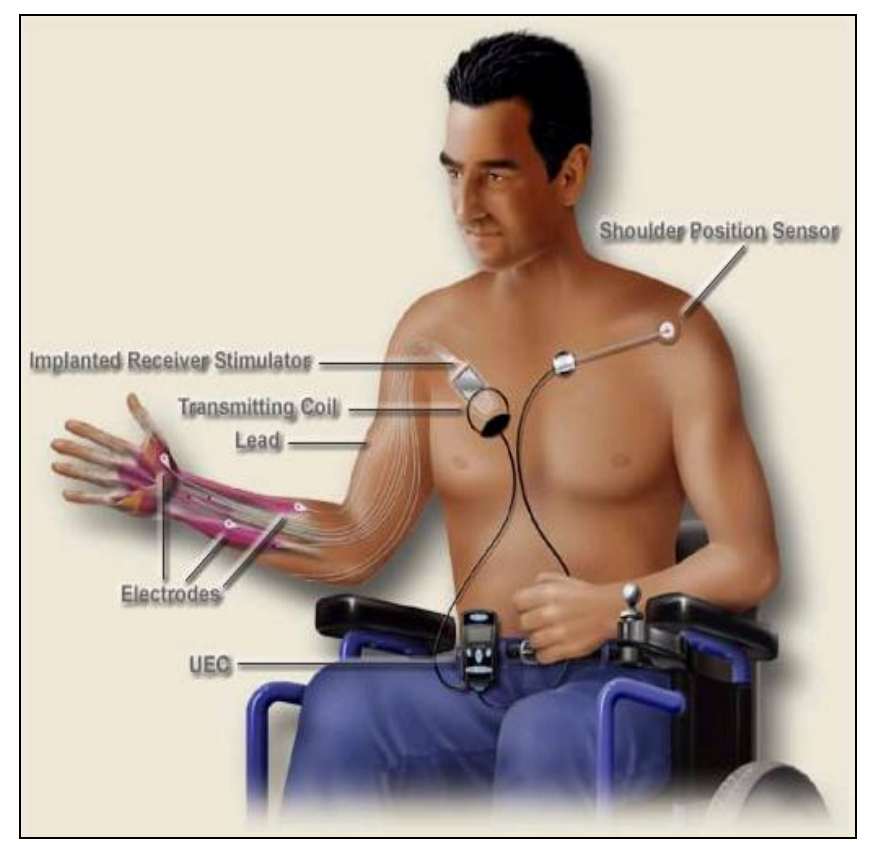

Figure 1: The Free Hand System by NeuroControl Corp. has 8 or more stimulating leads to restore function to the hand. It is triggered by a shoulder movement.

*Chief Scientist at the Alfred E. Mann Foundation for Biomedical Engineering

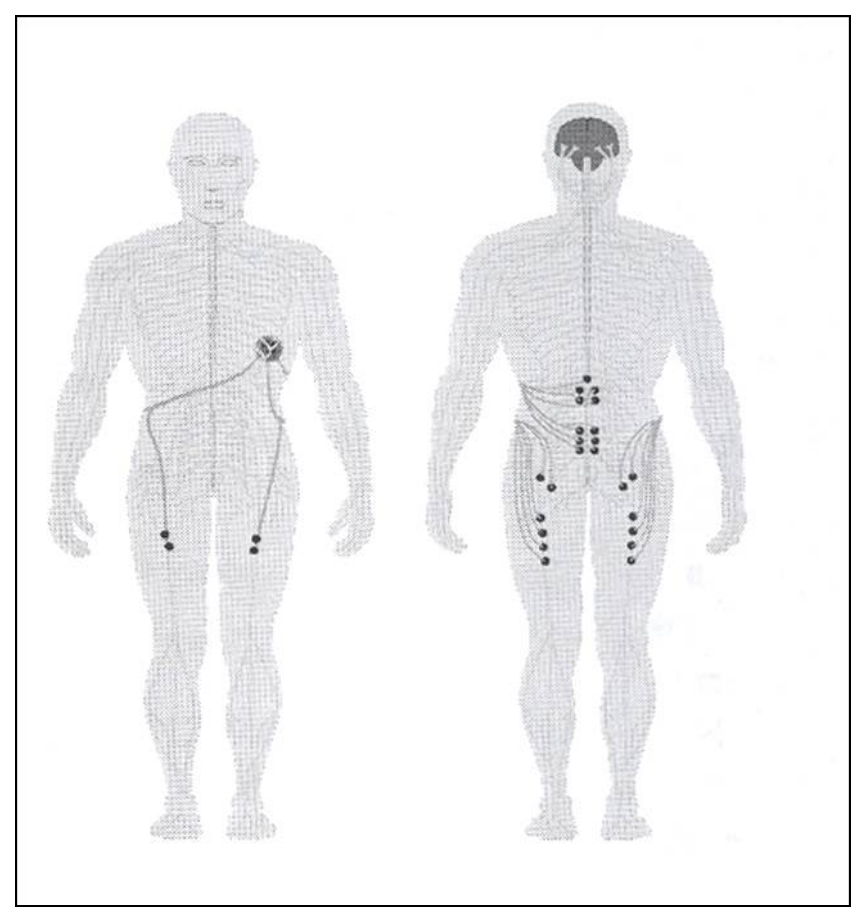

Figure 1A: The Nucleous FES 22 by Neopraxis Pty Ltd has 22 stimulating leads. It was designed to restore partial function to the legs and provide some urinary control.

The problems associated with these and other multi-leaded Functional Electrical Stimulation (FES) systems are:

1. Extensive surgery requiring on the order of 4 or more hours, and in some cases several days of surgery to tunnel long wires thru the body, attach the different electrode terminations onto the correct anatomical locations of the targeted nerves and muscles, and make the pocket for the implantable device.

2. Threat of infection: The surfaces of the wires, electrodes and devices seem to protect infectious microorganisms from the body's immune system. By the time a bacterial infection is detected, it is always assumed that the entire interconnected system has become contaminated. This requires that the entire implant system be surgically removed. In some cases the explant procedure is more difficult than the implant surgery and can take longer. There is also always the danger for serious damage to the patient from the explant procedure.

3. External coil orientation and position: External magnetic powering requires a coil to be worn by the patient in close proximity to the implanted device, and to be oriented correctly. If the coil is displaced by a relatively small distance the entire system becomes intermittent or shuts down.

4. Significant advanced planning and inflexibility: The precise number and type of sensors, stimulators, lead lengths and device locations specific to each patient and 
his/her condition must be planned for in advance. Adding extra channels that were not planned for can become a major engineering project.

5. Coordination of sensors and stimulators and external wires: Many systems had implantable stimulator channels and external sensing channels. The external sensors required wires outside the body running between the sensor and the external powering/control unit, in addition to the wires from the coil.

As a result of these problems, multi-leaded multichannel implantable stimulator-sensing systems to provide daily functional support for paralyzed limbs have not been successfully commercialized. Only multichannel stimulators with all the electrodes on one or two cables such as spinal cord stimulators and cochlear implants that operate at a specific anatomical location have been successfully commercialized.

\section{A PRACTICAL MULTI-CHANNEL FUNCTIONAL ELECTRICAL STIMULATION SYSTEM}

A practical FES system needs to be wireless to prevent the entire system from getting infected. The body's immune system is quite effective. Foreign objects provide a partial shield over which the infection can migrate. If different components of an implantable system were not connected, the probability that an infection of one the components could migrate to another would be significantly reduced. In 1989 NIH funded the development of a magnetically powered wireless injectable stimulator. Three glass encased microstimulators were demonstrated to the NIH in 1982 and shown to be independently programmable. (See Fig. 2A). The stimulation energy and timing of up to 255 of these microstimulators could be independently controlled and powered thru a single magnetic field. Thus the infection problem was dealt with. If one microstimulator got infected, there was a high probability that the infected device could be removed without infecting the others.. This design was later improved with a more efficient assembly (Fig. 2B) and a strong ceramic case with eyelet (Fig. 2C). Figures 3 and 4 show $\mathrm{x}$-rays with five devices which were injected to stimulate certain nerves. When stimulated, these nerves could move the arm, thumb, fingers, and wrist of a stroke patient. The photo on the right is a demonstration of the control unit and the coils mounted on an arm to power and control the five injected micro-stimulators.

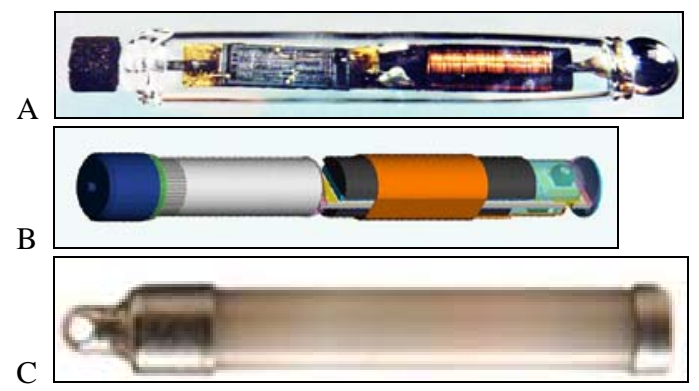

Fig 2: A) Glass microstimulator1992, left to right: Tantalum oxide anodic-electrode-capacitor, integrated circuit (IC), and ferrite wound coil. B) Internal components, electrode, capacitor, and coil wound on ferrite sandwiched IC. C) Advanced design in a hermetic ceramic case.

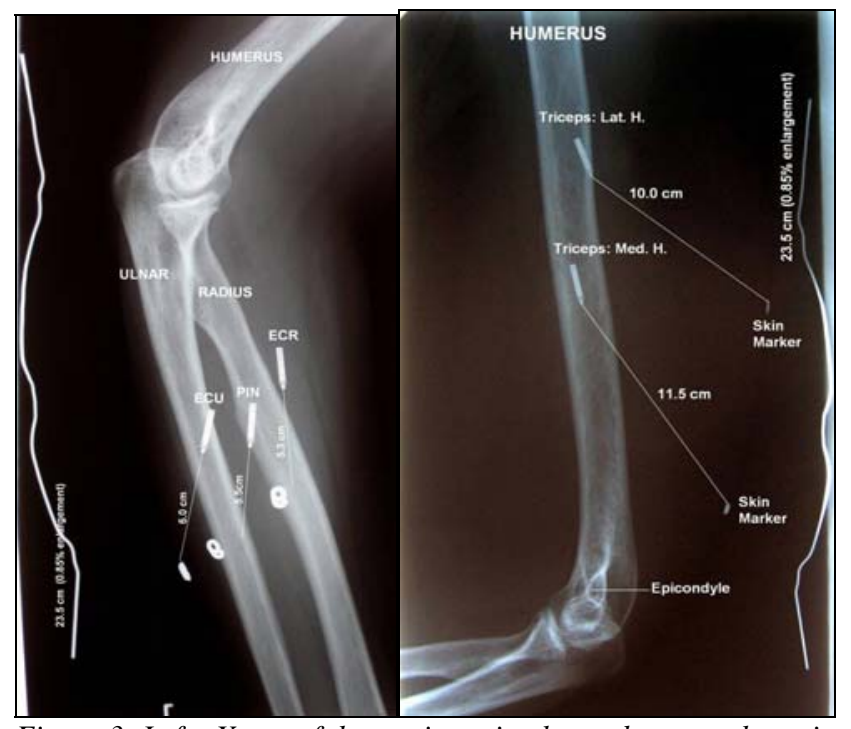

Figure 3: Left: X-ray of three microstimulators between the wrist and elbow positioned to stimulate the nerves that cause the fingers to open, the thumb to rise, and the wrist to move upward. Right $x$-ray of the two microstimulators positioned between elbow and shoulder to stimulate the nerves to cause the arm to straighten out.

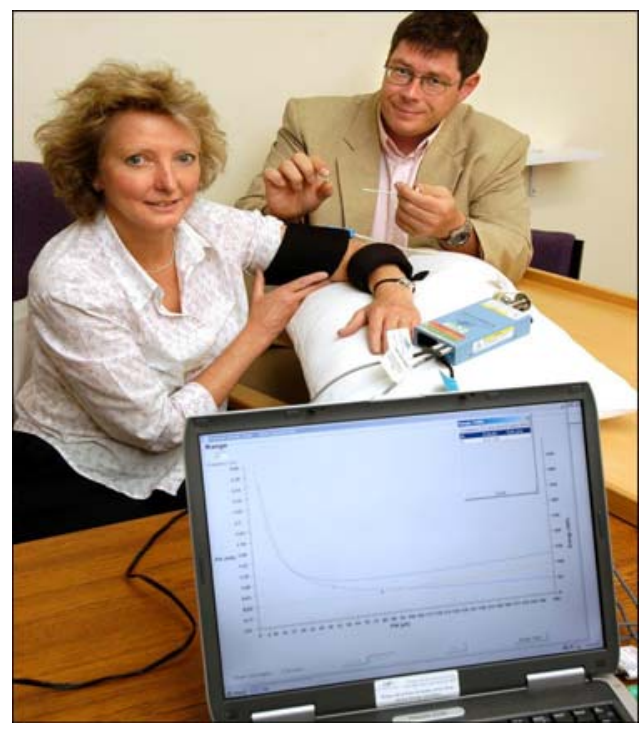

Figure 4: Demonstration of the coils (black) mounted on the arm to power and control the five microstimulators, and the blue control unit on the table next to the hand. The demonstrators are Dr. Jane Burridge of South Hampton England (the principal clinical investigator) and Dr. Gregoire Cosendai (The AMF Field Bioengineer).

These magnetically powered microstimulators satisfy problems 1 and 2 mentioned in the introduction above, i.e. the wireless requirement and the minimization of surgery. In clinical studies on six patients it was determined that with training, a surgeon can learn to inject these devices in the correct location at a rate of about 15 minutes per microdevice. However, we need microsensors, and have to get rid of the external coils and wires that power and control the microstimulators. This requires new technologies inside each of the micro devices, a battery, a propagated electromagnetic wave radio transceiver, and the addition of sensing technology. This also requires a small external 
computerized controller (master control unit or MCU) with a matching transceiver, initially outside the body since its program will have to be modified as we add microdevices, and later inside the body if a totally implanted system was desired.

\section{POWER SOURCE FOR EACH MICRODEVICE}

In about 1995 it was decided that the battery should be a hermetic $10 \mathrm{mWHr}$ Lithium Ion (LI) cylindrical cell. The LI cell has about five times the energy density of nickel cadmium batteries, and has excellent charge retention properties. In about 1998, a hermetic LI cell meeting our requirements was developed by Quallion Corp. [1] See Figure 5. This cell was about $2.5 \mathrm{~mm}$ in diameter and $12 \mathrm{~mm}$ long. It had a capacity of about $10 \mathrm{mWHrs}$ (3 mAHrs at about 3.6 volts).

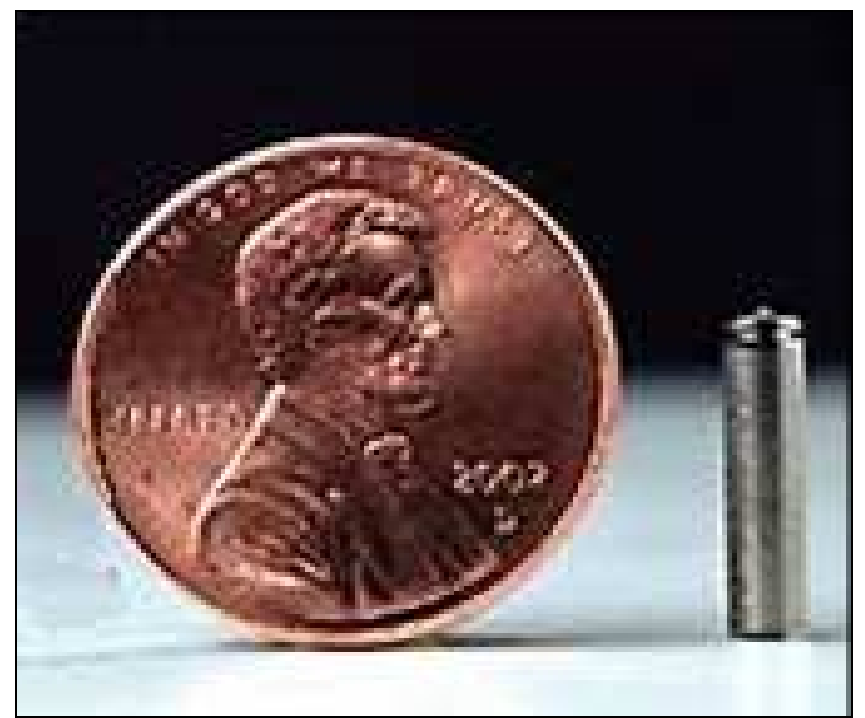

Figure 5. $10 \mathrm{mWHrs}$ Lithium Ion rechargeable battery that is about $2.7 \mathrm{~mm}$ in diameter and about $12 \mathrm{~mm}$ long.

\section{BATTERY CHARGING SYSTEM}

The wireless powering system to recharge the battery was similar to that used to power the glass microstimulator, (i.e. transformer coupling). The constraints however were less demanding. A small amount of intermittency and coils could be allowed as long as the battery was charged in a reasonably short time. The goal is one hour per week or five minutes per day. A ferrite tube was used for the core of the coil. Most of the electronics is located inside the ferrite tube. See Fig. 6 . The coil is about $2.5 \mathrm{cc}$ in diameter, and about $8 \mathrm{~mm}$ long. The charging coil is very large, (about two or more inches in diameter and several inches long), and thus there is negligible loading of the large coil's field by the microdevice's coil. By designing the large coil with a high "Q" and not having any modulation on the magnetic field, it is possible to efficiently generate a very large magnetic field with a small amount of power, and permit recharging microdevices deep inside the body (e.g. more than four inches inside the body). $125 \mathrm{Mhz}$ is used for the charging field.

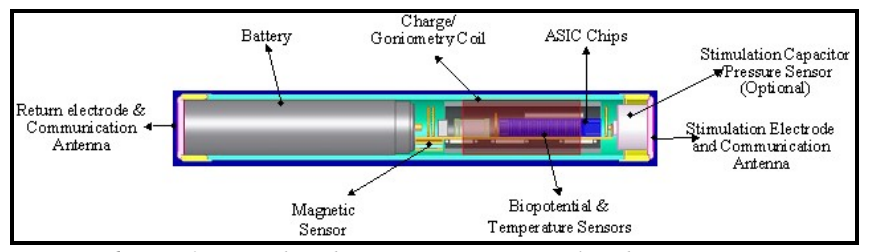

Figure 6: Left to right: battery, two vertical inductors, capacitor half in ferrite tube. The blue represents several integrated circuit chips, with a crystal on top inside the ferrite tube, and the output capacitor on the right.

\section{BIDIRECTIONAL TELEMETRY SYSTEM}

The requirement is a low power telemetry system, capable of communicating from any part of the human body to the MCU which could be 4 meters away on a nightstand.. The lowest power wireless scheme is to use propagated electromagnetic waves, i.e. radio signals. It has been estimated that if certain muscles in a persons leg were more than $50 \mathrm{mSec}$ late in responding while running, that person could fall down.[2] Also electrical noise could introduce an error in the message. Thus we need error detection codes, and need to be able to resend the message. Based on these situations, we decided to perform all communications within a $10 \mathrm{mSec}$ time frame. This time frame would allow us to repeat the same message five times before we were physiologically late. The data to each device requires the selection of one out of about a hundred parameters plus about 8 bits to implement a new value. The response would usually be an eight bit value for a preprogrammed parameter, plus two special bits. One special bit indicates the error check status of the previously received transmission, and the other special bit indicates whether or not an alerting condition was detected. The alerting condition could be low battery capacity, unsafe overcharging, an undesired temperature rise, etc. Thus in every frame 15 and 10 data bits plus an equal number of forward error detecting bits are transmitted to and from each micro device respectively.

\section{TRANSCEIVER COMMUNICATION POWER CONSIDERATIONS}

The first amplifier component (field effect transistor or current transistor) in a receiver front end for the band 400 to $460 \mathrm{MHz}$ needs a powering current of about one milliamp to be able to lower the noise floor to detect microvolt signals. A transmitter located 4 inches inside the body needs an equivalent radiated power (ERP) of about $1 \mathrm{~mW}$ (if it were in air) to get a radio signal out of the body that is reliably detectable 4 meters away by a sensitive receiver. The power to the transmitter output stage needs to be about three times the ERP. This means that about $1 \mathrm{~mA}$ at 3.5 volts is needed for the transmitter.

Continuous reception is out of the question since the $3 \mathrm{mAHr}$ battery would be drained in 3 hours with a continuous $1 \mathrm{~mA}$ drain.. Besides the transceiver power requirement, we also need the battery power to do jobs like stimulating nerves and sensing various parameters. The solution we came up with is to have a very short transmit and receive duty cycle (about $0.1 \%$ ) that is accurately synchronized. For each $10 \mathrm{mSec}$ frame the implant would receive for $5 \mu \mathrm{Sec}$, and then at a later time in the frame transmit for $5 \mu \mathrm{Sec}$. With a $0.1 \%$ duty cycle one $\mathrm{mA}$ of power drain becomes an average of one $\mu$ A.of power drain. This scheme requires a clock accurate to a fraction of a microsecond every ten $\mathrm{mSec}$. The receiver also has to be designed to start up quickly.

If the total transmit and receive time is only ten microseconds, this also means that the transmitter and receiver is off for 9,990 $\mu \mathrm{S}$. Thus another device can transmit and receive during the off time. 
In fact if the timing were very exact, 999 other devices can communicate during the off time. In reality we were restricted to about 850 devices during the off time.

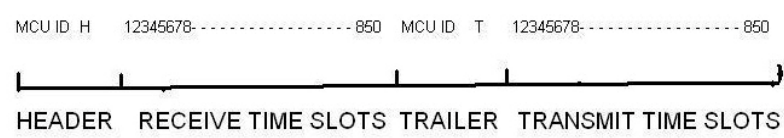

Figure 7: Upload and download timing diagram: The header, trailer, and time slots are shown for a single frame.

\section{SYNCHRONIZING TO TIME SLOTS}

The MCU contains a microprocessor, an accurate clock, and a transceiver designed to communicate with each of the microdevices used in the system. At time of implant, each micro device will be assigned a time slot, and the ID of its MCU. Time slots will be assigned in numerical sequence. The MCU has a program that determines the coordination of all of its microdevices. At the beginning of each frame the MCU broadcasts a header with 11 time slots, and half way into the frame it also transmits a trailer involving 11 time slots. The header contains the MCU ID and other information. When the microdevice initially turns on it starts looking for the header or trailer. After it locates the start of the header, it synchronizes its clock to that of the MCU, and then locates its own time slot. From that point onward, it only listens to its own time slot, and continues to synchronize its own clock using the time slot information. Fig. 7 is a diagram showing the header, time slots, and trailer.

\section{SENSORS}

In the initial versions, we intend to have two programmable sensor features, voltage, and distance measurement for joint angles.

\section{VOLTAGE AMPLIFIER}

A programmable voltage amplifier capable of detecting muscle or neural potentials. Signals between $3 \mu \mathrm{V}$ and $0.1 \mathrm{~V}$ with 30,000 sample/second resolution. The voltage signal is analyzed during each frame, and either the number of nerve spikes or muscle potentials are transmitted back for each frame, or a rectify and integrate circuit indicates the average amplitude of all the signals in a single frame. During fitting, the clinician can have an oscilloscopic view of the signal at 30,000 samples/second, and decides which analysis parameters he wishes to set up.

\section{GONIOMETRY}

The pickup coil for charging the battery is used as a $125 \mathrm{kHz}$ magnetic field transmitter in one microdevice, and as a magnetic field strength detector in another microdevice. The distance between a transmitting and receiving device can be determined within a few percent accuracy between one and twenty centimeters by the strength of the received signal. A reading is sent every frame. This is useful in monitoring and controlling the opening and closing of a limb joint.

\section{TEMPERATURE}

Temperature is also monitored for engineering purposes. This is obtained by amplifying the thermal coefficient of a transistor base-emitter junction. It is accurate to about 0.2 degrees centigrade. A block diagram of the circuit is shown in Fig. 8 and the system diagram is shown in Fig. 9.

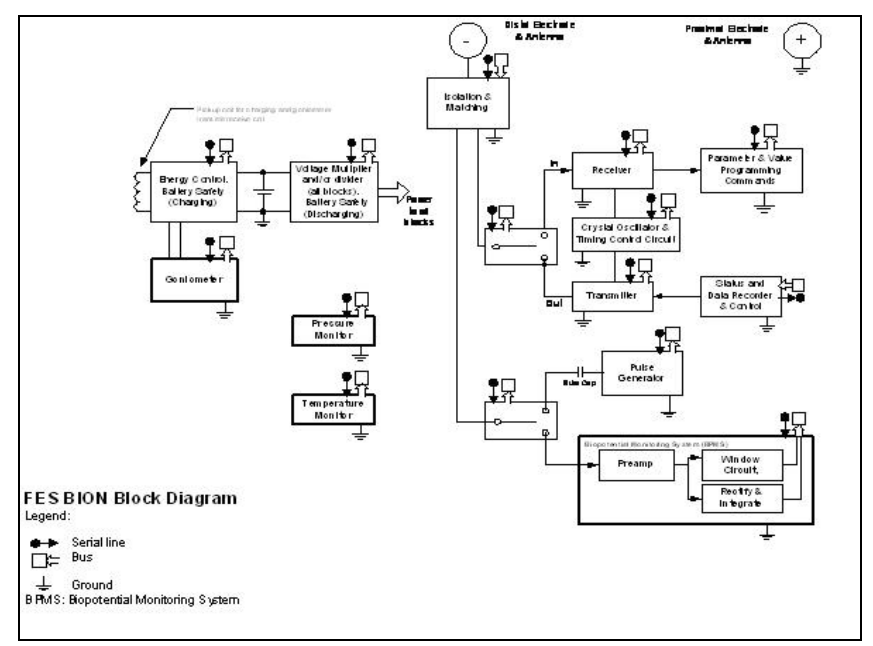

Figure 8: Block diagram of implantable microdevice.

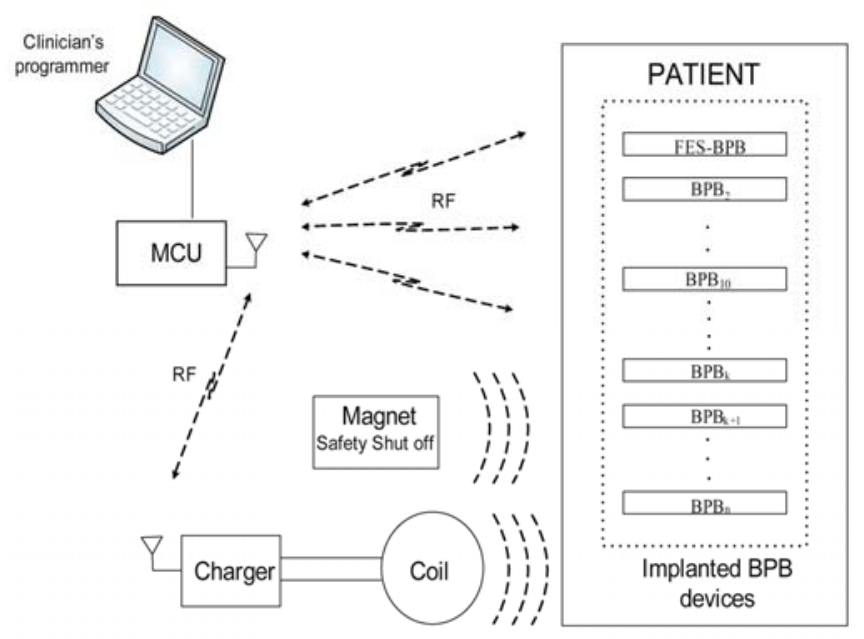

Figure 9: SYSTEM DIAGRAM: The magnet safety shut off is an optional feature in case the microdevices are used in a stand alone mode. In that situation, the only safe stimulation shut off is to bring a permanent magnet near the implant. If the MCU is used, the implants can be programmed to stop stimulating if radio signals stop being received for a predetermined time

At the present time the project is close to releasing the final integrated circuits for the first version.

\section{ACKNOWLEDGEMENTS}

I wish to thank Phil Troyk and Jerry Loeb for bringing me into this project. I also wish to thank Al Mann and the staff at the Alfred Mann Foundation for the great work in bringing these devices to fruitation. Partial funding for this project came from the U.S. National Institute of Health under contact No. 1-NS-2-2322.

\section{REFERENCES}

[1] About 1995 the AMF made a major effort to obtain such a battery from each of the three best quality major international battery manufacturers. The small size of the medical market compared to the large cell phone and computer markets prevented these companies from having an economically feasible relationship with us. In the end Alfred Mann personally founded a company, "Quallion” specifically to make these high reliable Li-I batteries.

[2] Private communication from Dr. Don McNeal when he was at Rancho Los Amigos Hospital 